\title{
Article \\ Selection of Fuel Isotope Composition in Heating Injectors of the FNS-ST Compact Fusion Neutron Source
}

\author{
Sergey Ananyev*, Alexey Dnestrovskij ${ }^{(1)}$ and Andrei Kukushkin \\ National Research Center Kurchatov Institute, 123182 Moscow, Russia; Dnestrov0@gmail.com (A.D.); \\ ank755@gmail.com (A.K.) \\ * Correspondence: Ananyev_SS@nrcki.ru
}

Citation: Ananyev, S.; Dnestrovskij, A.; Kukushkin, A. Selection of Fuel Isotope Composition in Heating Injectors of the FNS-ST Compact Fusion Neutron Source. Appl. Sci. 2021, 11, 7565. https://doi.org/ 10.3390/app11167565

Academic Editor: Arkady Serikov

Received: 1 July 2021

Accepted: 16 August 2021

Published: 18 August 2021

Corrected: 28 September 2022

Publisher's Note: MDPI stays neutral with regard to jurisdictional claims in published maps and institutional affiliations.

Copyright: (C) 2021 by the authors. Licensee MDPI, Basel, Switzerland. This article is an open access article distributed under the terms and conditions of the Creative Commons Attribution (CC BY) license (https:// creativecommons.org/licenses/by/ $4.0 /)$.

\begin{abstract}
For the FNS-ST compact neutron source, the dependence of the neutron yield on the tritium content in the bulk plasma is analyzed for the operation of the heating injectors with different isotope compositions of the neutral beams. Self-consistent simulations of the FNS-ST operating regimes are performed using the SOLPS4.3 and ASTRA codes for different densities of the bulk plasma and diffusion coefficients. The FC-FNS code is used to calculate the required fluxes of the fuel components into the plasma provided by different injection systems: the pellet injectors and the neutral beams. In simulations, the plasma density is varied in the range $n_{e}=(7-10) \times 10^{19} \mathrm{~m}^{-3}$, and the ratio of the diffusivity to the heat conductivity in the range $D / \chi_{e}=0.2-0.6$. For the scenarios with the $\mathrm{D}+\mathrm{T}$ or $\mathrm{D}$ beams, in the window of the operating parameters, the maximum possible fractions of tritium in the bulk plasma are calculated, and the corresponding neutron yields are obtained. For the regimes with the maximum neutron yield $(4.5-5.5) \times 10^{17} \mathrm{~s}^{-1}$, the accumulation of tritium at the site (up to $550 \mathrm{~g}$ ) is calculated for different heating beams.
\end{abstract}

Keywords: fusion neutron source; FNS-ST; spherical tokamak; neutral beams; hydrogen isotopes; fuel cycle; tritium accumulation; neutron intensity; self-consistent simulations of plasma

\section{Introduction}

In the FNS-ST fusion neutron source [1,2] based on a spherical tokamak that provides plasma with fusion parameters, the reaction between tritium and deuterium ions will proceed, in which helium ions and fast neutrons will be produced, while the tritium and deuterium consumed in the reaction must be refilled. For feeding the plasma, the $\mathrm{D}_{2}$ and $\mathrm{T}_{2}$ fuel isotopes can be injected into the plasma in the form of frozen pellets. Another way of feeding the plasma is the injection of T or D neutral beams. Such injection will make it possible to solve several problems: to provide additional plasma heating, to drive the current, and to fuel plasma with isotopes of hydrogen.

In [1-4], it was proposed to use D + T plasma with a 1:1 isotope composition (that is, the fraction of tritium in the plasma is $f_{\text {core }}{ }^{T}=0.5$ ) and to use the injection of neutral beams with similar isotope composition. Meanwhile, in the FNS-ST compact tokamak, the fusion reaction will proceed mainly due to the interaction of the beam-produced fast ions with the plasma $\left(P_{f(b p)}\right)$ [5]. We will consider two approaches to the design of the heating injectors and fuel cycle (FC) of the FNS-ST facility: a D + T beam (with the composition close to 50/50) and a pure deuterium beam.

For the compact FNS-ST tokamak, the flux of the particles supplied by the beam is comparable to that required for fueling the bulk plasma. Thus, the balance of particles (D and T) must be ensured in the bulk plasma when using the D or D + T beams and injecting the $\mathrm{D}$ or $\mathrm{T}$ fuel pellets. In this case, to increase the neutron yield, it is reasonable to increase the fraction of tritium in the plasma. In this article, the consistent solution to this problem is considered. 


\section{Approach to Organizing the FNS-ST Fuel Cycle}

According to [6,7], designing the FNS-ST facility will be a step toward the development of the stationarily operating DEMO-FNS neutron source [8,9] and the development of some technologies, including fusion FC technology [10]. The FNS-ST will be a stationary operating facility, and its FC structure will be similar to that of the DEMO-FNS [11,12], with allowance for the specific features of the facility. The fuel cycle of the FNS-ST includes systems for storing the fuel isotopes, pumping the gases from the vacuum chamber, separating the hydrogen isotopes from the mixed pumped-out gases, additionally purifying the chemically bonded hydrogen isotopes, processing the tritium-containing radioactive waste (including the system for removing tritium from the gas-water mixture), producing tritium and extracting it from the carrier gas, separating the hydrogen isotopes to obtain their concentration required to supply the injection systems, as well as the control systems for the sources of hydrogen isotopes in the plasma and the heating and fuel supply systems. In this design stage, we do not consider the possible systems providing circulation of impurity gases (for injecting them into the divertor) or lithium circulation on the first wall, since their effect on the FC operation is moderate. They will be considered in the next design stages. In this work, we calculate the particle fluxes in the FC systems to determine their efficiencies. Optimization of the FC systems will be performed in the future based on the calculations carried out in this work.

At first, for the FNS-ST project, it was planned to use the off-axis injection of fast atoms with the slanted arrangement of the injectors [1,2]. Subsequent analysis $[13,14]$ showed that it is technically difficult to realize slanted injection, and it is not reasonable to tilt the injection axis off the equatorial plane. It was also proposed to inject deuterium beams since the injection of tritium in the form of fast atomic beams creates some technical difficulties. Similar to the DEMO-FNS simulations [11,15], in this work, we calculate the scenarios for using the $\mathrm{D}+\mathrm{T}$ or $\mathrm{D}$ heating beams. In this case, the calculations are performed relying on injectors based on the positive ion source, which results in the appearance of the beam components with lower energies: $\mathrm{E} / 2$ and $\mathrm{E} / 3$.

The calculations based on the consistent model of the bulk and divertor plasmas performed for the DEMO-TIN $[15,16]$ showed that to maintain the required plasma density, it was insufficient to fuel the bulk plasma with the beams and neutral flows from the divertor. As a result, it became necessary to use pellet injection along with large-scale gas injection. Since for the spherical tokamak, it is difficult to inject the fuel pellets from the high magnetic field side (HFS), the injection at the FNS-ST is foreseen from the low field side (LFS). This results in additional fuel losses due to the outward drift, and to maintain fuel balance, we should increase the fuel inflow with the pellet injection. This results in an increase in the required tritium reserve at the facility.

The project in $[8,17]$ provides for the hybrid blanket, but it does not provide for tritium breeding. In the simulations, we use a tritium breeding ratio (TBR) equal to 1.25 , which is sufficient to replenish the tritium burnout in the core plasma. The FNS-ST parameters are discussed in detail in the next sections.

\section{Possibility of Injecting Tritium with Fast Atomic Beams}

For the FNS-ST project, the heating beams with different isotope compositions can be developed based on either positive or negative ions, which are accelerated to the required energy and subsequently neutralized in the gas target. This is possible due to the transitional range of atomic energies required for the most efficient current drive and heating of the FNS-ST plasma [13]. In [14], both options for the beam formation were analyzed, and it was recommended to form the neutral beam from the positive ions.

In the "classical" neutral beam injector based on the positive ions, the beam source is connected directly to the gas neutralizer. The same hydrogen isotope is used for beam neutralization and formation, and the initial gas is also composed of it. For the T or D $+\mathrm{T}$ injector, this operating principle can also be used. However, the option of the use in the neutralizer of gas with the isotopic ratio different from that in the source should be 
considered to reduce the amount of $T_{2}$ in the gas supply system of the injector and FC of the facility (see, for example, [18]). The gas flow injected into the ion source must ensure the production of a sufficient amount of deuterium and tritium ions to feed the bulk plasma. In this case, it is not necessary to use $\mathrm{T}_{2}$ as the gas forming the neutralizing target. Both pure $\mathrm{D}_{2}$ and a $\mathrm{D}+\mathrm{T}$ mixture could be used there to simplify the system for gas treatment in the closed or open general fuel cycle (FC) of the injection system. At the same time, in the systems for gas supplying, pumping, and processing (the isotope separation system), the amount of tritium should also be minimized to meet the basic safety requirements.

The design of the injector operating with a $\mathrm{D}+\mathrm{T}$ mixture should be optimized to deliver the required amount of $\mathrm{D}^{0}+\mathrm{T}^{0}$ to the bulk plasma with the minimum amount of $\mathrm{T}_{2}$ stored in the injector and gas supply system. To maximize the injection of $\mathrm{T}^{0}$, the ion source current should be maximal, and it is necessary to provide the required isotope composition of the accelerated beam. In addition, the efficient neutralization of ions and efficient atom transportation along the beam path must be ensured.

A distinctive feature of the beam injector based on the positive ions is the presence of the $\mathrm{D}_{2}{ }^{+}(15 \div 20 \%)$ and $\mathrm{D}_{3}{ }^{+}(7 \div 10 \%)$ molecular ions in the ion beam produced in the source. After dissociation and neutralization in the neutralizer, these ions produce atoms with energies $E / 2$ and $E / 3$ (in addition to the atoms with energy $E$ ). In the case of the $\mathrm{D}+\mathrm{T}$ injectors, the $\mathrm{T}^{+}, \mathrm{T}_{2}{ }^{+}, \mathrm{T}_{3}{ }^{+}, \mathrm{D}^{+}, \mathrm{D}_{2}{ }^{+}$, and $\mathrm{D}_{3}{ }^{+}$ions leave the source and accelerate. Consequently, the heating beam will contain the deuterium and tritium atoms with energies $\mathrm{E}, \mathrm{E} / 2$, and $\mathrm{E} / 3$. If we assume that the DT molecules are present in the working gas, then the $\mathrm{D}(2 \mathrm{E} / 5)$ and $\mathrm{T}(3 \mathrm{E} / 5)$ components will occur.

In the FNS-ST project, it is supposed that an additional power of $10 \mathrm{MW}$ can be input into the plasma, while in the basic operating regimes, the input power will be lower (6 MW). Such powers can be delivered using three 3.5 MW injectors. The operating scenario for the injectors is as follows: two injectors operate simultaneously, while the third one is in the regeneration mode. Increasing the additional heating power will require the use of four such injectors. The duration of the continuous injector operation is limited by the explosion safety condition. In the course of pumping out the gas entering the injector chamber, the gas becomes frozen on the cryo-panels, and its amount can reach a critical value for which, in the event of the emergency breakthrough of atmospheric air into the vacuum chamber and rapid warming of the panels in the injector volume, an explosive mixture can form. For the required gas flux of $6.3 \mathrm{~Pa} \cdot \mathrm{m}^{3} / \mathrm{s}$ [14], the injector operation should be terminated after $2.2 \mathrm{~h}$ of operation to regenerate the cryo-panels. This criterion terminates the injector operation before another condition becomes satisfied: the excess of the allowed total amount of $T_{2}$ contained in one room (injector) [19].

\section{Consistent Model for Calculating Fluxes in the Fuel Cycle}

The FC-FNS code $[15,20,21]$ is used to calculate the fluxes of the fuel components in the FNS-ST fuel cycle and the accumulation of tritium in the FC systems. The code is adopted for calculating the FNS-ST parameters. It uses the data on the particle fluxes in the plasma, calculated with the SOLPS4.3 [16,22] and ASTRA [23] codes using builtin models of the bulk and divertor plasmas. The SOLPS4.3 code package realizes a 2D model of the edge (SOL and divertors) plasma in the assumption of the toroidal symmetry, involving the fluid description of the electrons and ions and the Monte Carlo modeling of the atoms and molecules. In the ASTRA code, a 1.5D approximation is used-that is, the one-dimensional (radial) transport of energy, particles, and current is modeled using the metric coefficients calculated from the 2D plasma equilibrium consistent with the plasma pressure and current profiles. The SOLPS run results relevant for the core-edge coupling are parameterized to form scalings for the plasma parameters at the separatrix, and these scalings form the boundary conditions for ASTRA [24]. This approach was previously used for analyzing the FC of the DEMO-FNS facility $[16,25,26]$, and in this work, it is used for the FNS-ST facility. To describe adequately in ASTRA the heat and particle fluxes in the bulk plasma, we use the Bohm/gyro-Bohm transport model [27] that takes into account the 
effects of nonlocal transport. This model developed for JET was successfully implemented for spherical tokamaks such as START [28] and MAST [29]. The diffusivity of the plasma particles and the average plasma density are varied to determine the possible range of variation of the fluxes in the FC. The particle diffusivity is taken to be proportional to the electron heat conductivity and is varied in the range of $D / \chi_{e}=0.2-0.6$ according to the research done on different devices $[30,31]$. The average plasma density is varied inside the range of $n_{e}=7.0-10.0 \times 10^{19} \mathrm{~m}^{-3}$, which, together with the variation of the diffusivity, determines the possible range of the fluxes in the FC. The plasma density profile is then determined by the electron heat conductivity together with the particle sources from the gas influx across the separatrix, pellet injection, and NBI. The gas influx is calculated in the SOLPS code, and the profile of the corresponding ion source is calculated in ASTRA and depends on the density and temperature profiles inside the separatrix. The radial profile of the ion source from the pellet injection is pre-defined, with the maximum at some distance from the separatrix and the magnitude as the external parameter. The radial profile of the ion source from the neutral beam is calculated with the NUBEAM code [32] built in ASTRA. An example of the resulting density profile for a $\mathrm{D}$ beam case is shown in Figure 1 . No density profile difference between the $\mathrm{D}$ and $\mathrm{D}+\mathrm{T}$ beam cases is observed in our calculations. Note that the density profile shape has a minor impact on the neutron yield that is dominated by the beam-plasma interaction.

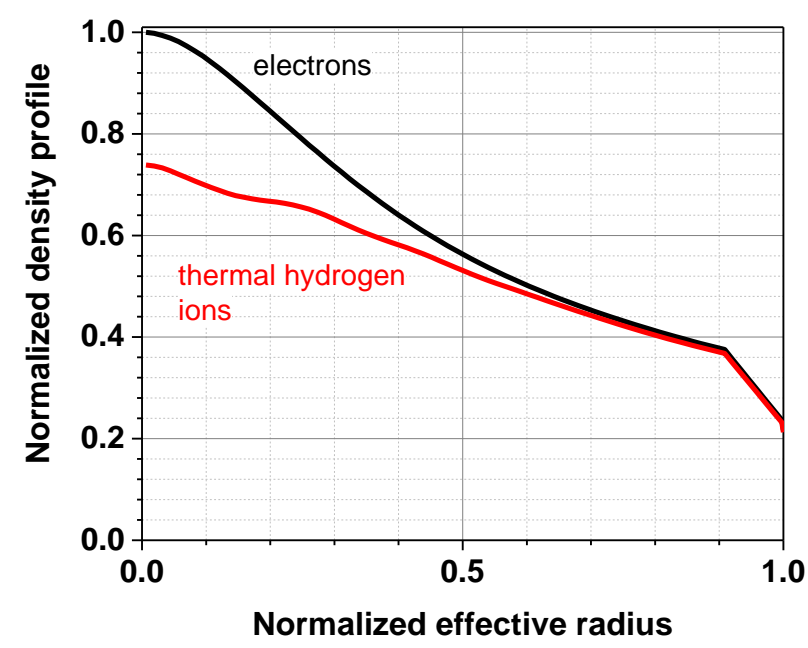

Figure 1. The electron and thermal hydrogen density profiles normalized to the central electron density for a $\mathrm{D}$ beam case.

The resulting dimensionless plasma parameters, such as the collisionality of $\sim 0.02$ and the normalized ion Larmor radius of $\sim 0.03-0.04$ in FNS-ST (with the central ion temperature of 6-8 keV), are not far from those in the shots from the database analyzed in [30,31], thus justifying the implementation of this transport model for FNS-ST.

We use the FC-FNS code developed at the NRC Kurchatov Institute (Russian Federation) to simulate the balance of particles in the core and divertor plasma (based on the results calculated by the ASTRA and SOLPS codes) as well as the flows in all fuel cycle systems (in steady-state mode) and, accordingly, the calculation of the $\mathrm{T}$ inventory in them. To calculate the $\mathrm{T}$ inventories at the facility site, the values are calculated for all fuel cycle systems (based on the physical principles of their operation, the calculated isotope fluxes, and their concentrations), including the starting storage (containing $\mathrm{T}$ at the time $\mathrm{T}$ breeding systems are stopped). Since no extended T breeding is planned at the facility, the "T inventory" characterizes the value both at the moment of starting the facility and at any other moment in time (not containing the time-integrated $\mathrm{T}$ accumulation value).

The FNS-ST parameters used for the FC simulations are shown in Table 1. The calculations show that the neutron yield varies in the range expected for the project [1]. 
Optimization of the hybrid blanket to obtain the required TBR at the corresponding neutron intensities is beyond the scope of this work and will be performed in the future.

Table 1. The FNS-ST parameters used in the FC simulations.

\begin{tabular}{cc}
\hline Parameter & Value \\
\hline Major plasma radius $R(\mathrm{~m})$ & 0.5 \\
Ratio $R / a$ & 1.66 \\
Elongation $\kappa_{\text {elong }}$ & 2.75 \\
Triangularity $\delta$ & 0.5 \\
Plasma current $I_{p}(\mathrm{MA})$ & $1.25-1.5$ \\
Non-inductive current, fraction & 1 \\
Magnetic field at the geometrical axis $B_{t}(\mathrm{~T})$ & 1.5 \\
Volume-averaged electron density $<n>\left(10^{20} \mathrm{~m}^{-3}\right)$ & $0.7-1.0$ \\
Total neutron yield $S_{\text {neut }}\left(\mathrm{s}^{-1}\right)$ & $3-6 \times 10^{17}$ \\
Neutron load onto the first wall $(\mathrm{FW})\left(\mathrm{MW} \cdot \mathrm{m}^{-2}\right)$ & 0.2 \\
Beam energy $E_{b}(\mathrm{keV})$ & $130-140$ \\
Beam heating power $P_{N B I}(\mathrm{MW})$ & $6-10$ \\
Electron cyclotron heating power $P_{E C}(\mathrm{MW})$ & $1-5$ \\
$H=\tau_{E} / \tau_{I P B 98}(y, 2)$ factor & $1.4-1.6$ \\
Normalized $\beta_{N}$ & $4-5$ \\
\hline
\end{tabular}

The NUBEAM-ASTRA calculations show that for both the $\mathrm{D}+\mathrm{T}$ and $\mathrm{D}$ beams, an increase in the tritium fraction in the core plasma $f_{\text {core }}$ is beneficial for the neutron yield $S_{\text {neut }}$. The simulation results are shown in Figure 2 . In the calculations with varying $f_{\text {core }}$, the isotope composition of the $\mathrm{D}+\mathrm{T}$ beam was taken unchanged such that $f_{N B}{ }^{T}=0.5$.

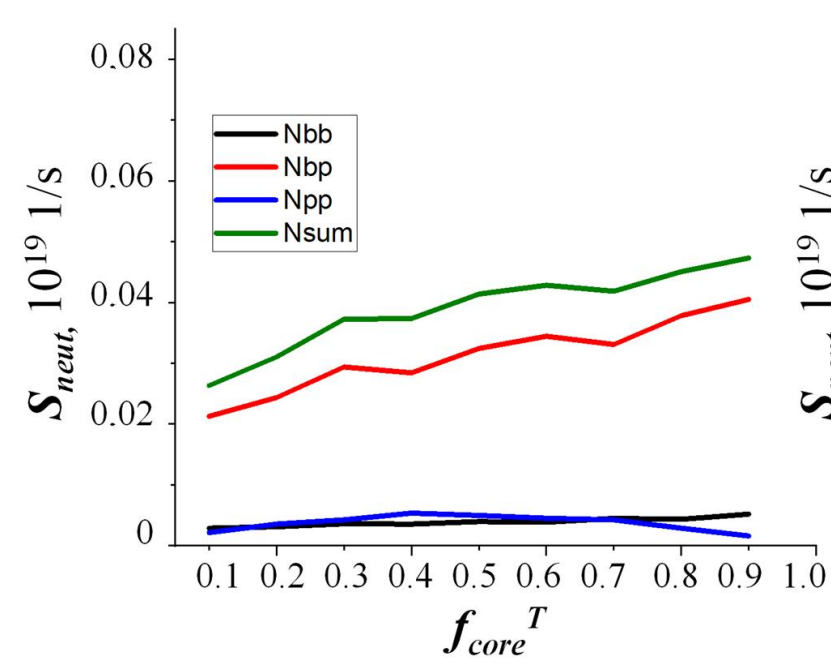

(a)

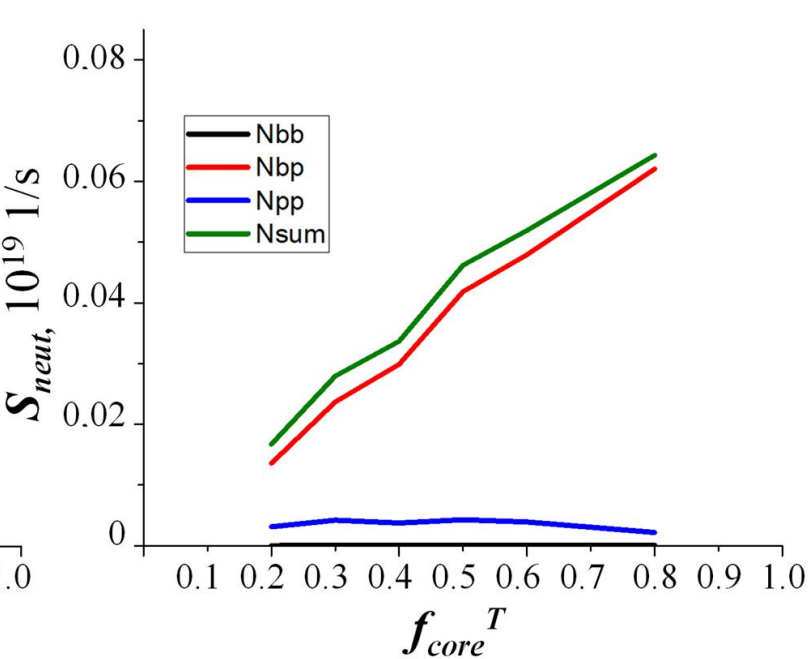

(b)

Figure 2. $D / \chi_{e}=0.4$ and $n_{e}=8.5 \times 10^{20} \mathrm{~m}^{-3}$. The neutron intensity $S_{\text {neut }}$ is a function of the fraction of tritium $f_{\text {core }}{ }^{T}$ in the plasma for the D $+\mathrm{T}(\mathbf{a})$ and $\mathrm{D}(\mathbf{b})$ beams. Different colors correspond to the neutron intensity components contributing to the total neutron intensity $S_{\text {sum }}(-)$. $S_{b p}(-)$ is the intensity due to fast ion interaction with plasma, $S_{p p}(-)$ is the intensity due to the plasma-plasma fusion mechanism, and $S_{b b}(-)$ is the intensity due to interaction between the fast ions.

In the FC-FNS code, the particle balance is described in the zero-dimensional approximation using the $S_{N B}, S_{p e l}$, and $S_{s e p}$ sources of the particles, assuming different lifetimes correlated with the ASTRA simulations [25].

\section{Discussion of Results}

In Figure 2, we can see that for the D + T beam, with an increasing fraction of tritium $f_{\text {core }}{ }^{T}$, the efficiency of neutron generation became higher. In the case of the $\mathrm{D}+\mathrm{T}$ beam, the 
DT reaction occurs as a result of two processes: the interaction of the fast deuterium ions with the thermal tritium (process 1 ) and the interaction of the fast tritium ions with the thermal deuterium (process 2). In this case, an increase in the neutron yield of the reaction of the beam-plasma interaction (Figure 2a, $N_{b p}$ ) with an increasing fraction of tritium implies that the contribution of process 1 to the DT reaction considerably exceeds that of process 2. Note that when the fraction of tritium in the plasma is $f_{\text {core }}{ }^{T}>0.5$ and the D beam is used, the neutron yield turns out to be higher (Figure 2b). For both types of the beam, the fraction of tritium in the plasma $f_{\text {core }}{ }^{T}$ can be different in different operating regimes (within the operating window of the $n_{e}$ and $D / \chi_{e}$ parameters) due to the considerable effect of the deuterium particle influx from the heating injectors on the isotope composition of the bulk plasma. For both types of the beam, the maximum possible neutron yield is reached at the maximum possible fraction of tritium in the plasma $f_{\text {core }}{ }^{T}(\max )$ (see Figure 2). In this case, the pellets injected into the plasma consist of pure tritium $\left(S_{p e l} D=0\right)$. The parameter $f_{\text {core }}{ }^{T}(\max )$ as a function of the $n_{e}$ and $D / \chi_{e}$ parameters is shown in Figure 3. For the D beam, in the ranges of low densities $n_{e}=7.0-9.0 \times 10^{19} \mathrm{~m}^{-3}$ and $D / \chi_{e}=0.2-0.3$, the small values of $f_{\text {core }}{ }^{T}(\max )$ can be explained by the low flux $S_{\text {pel }}$ and, therefore, by the considerable effect of the $S_{N B}{ }^{D}$ flux along with the $S_{\text {pel }}{ }^{T}$ flux. Within the operating windows of the $n_{e}$ and $D / \chi_{e}$ parameters, the maximum possible fractions of tritium are 0.85 and 0.7 for the $\mathrm{D}+\mathrm{T}\left(f_{N B}{ }^{T}=0.5\right)$ and $\mathrm{D}$ beams, respectively.

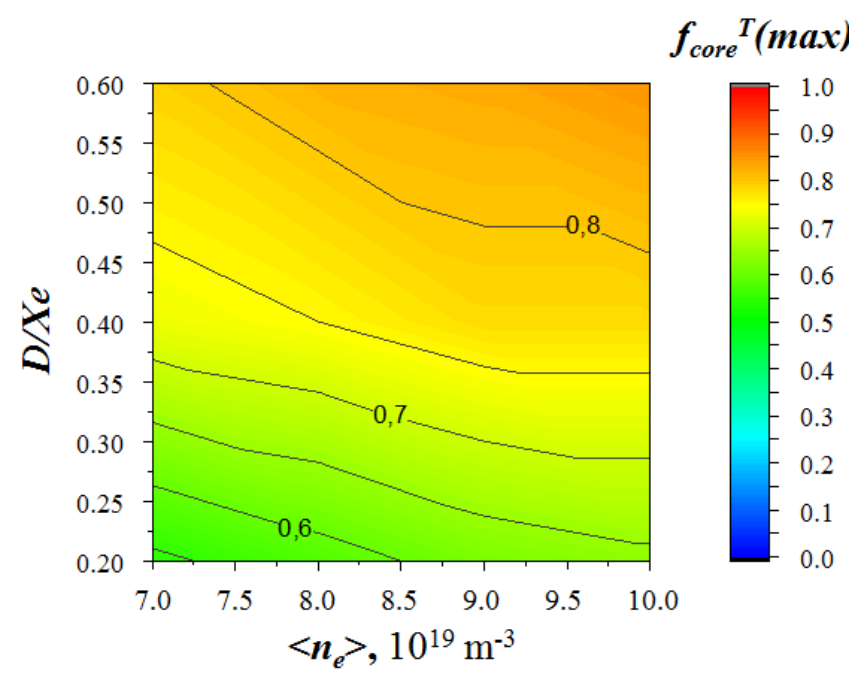

(a)

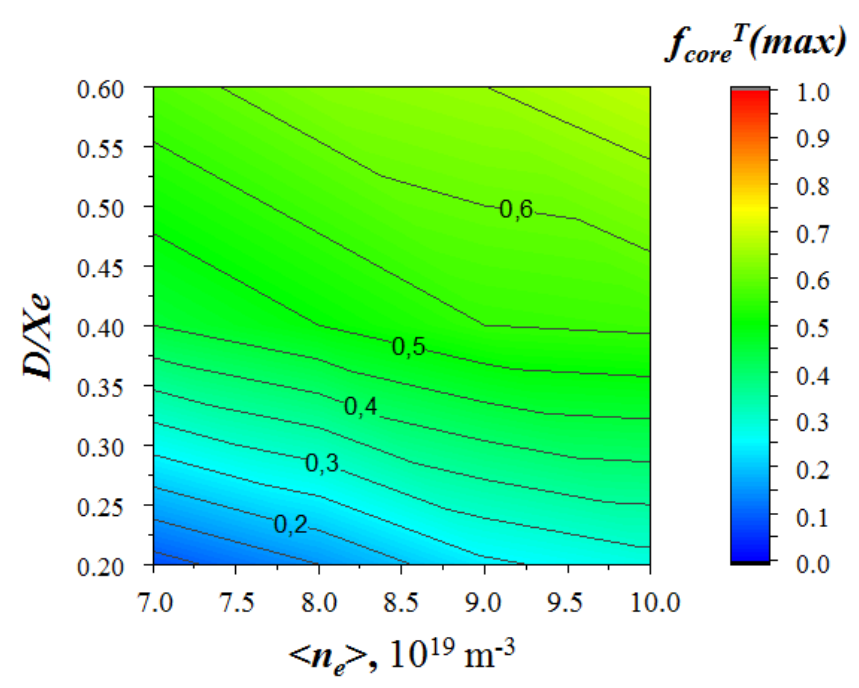

(b)

Figure 3. Maximum possible fraction of tritium $f_{\text {core }}{ }^{T}(\max )$ as a function of the $n_{e}$ and $D / \chi_{e}$ parameters for the (a) $\mathrm{D}+\mathrm{T}$ and (b) D beams. The scale on the right shows the correspondence of the color and $f_{\text {core }}{ }^{T}$ (max) values.

For the scenarios with the $\mathrm{D}+\mathrm{T}$ and $\mathrm{D}$ beams with identical plasma parameters $\left(n_{e}=8.0 \times 10^{19} \mathrm{~m}^{-3}\right.$ and $\left.D / \chi_{e}=0.4\right)$ but different fractions of tritium $f_{\text {core }}{ }^{T}(0.75$ and 0.5 , respectively), the absolute values of the $S_{N B}, S_{\text {pel }}$, and $S_{\text {sep }}$ fluxes are shown in Figure 4a. The contributions of these sources to the total number of particles, with allowance for different particle lifetimes for each source, are shown in Figure $4 \mathrm{~b}$. It can be seen that a rather large particle flux $S_{\text {sep }}$ from the divertor region (Figure 4a) makes a minor contribution to the total number of particles (Figure $4 \mathrm{~b}$ ). This can be explained by the peripheral localization of this source and, accordingly, by the short lifetime of the particles coming from it. In this case, in the range of the $n_{e}$ and $D / \chi_{e}$ parameters under consideration, the total flux $S_{p e l}{ }^{D}+S_{p e l}^{T}$ varies in the range of $\sim 10^{20}-10^{21} \mathrm{~s}^{-1}$. 


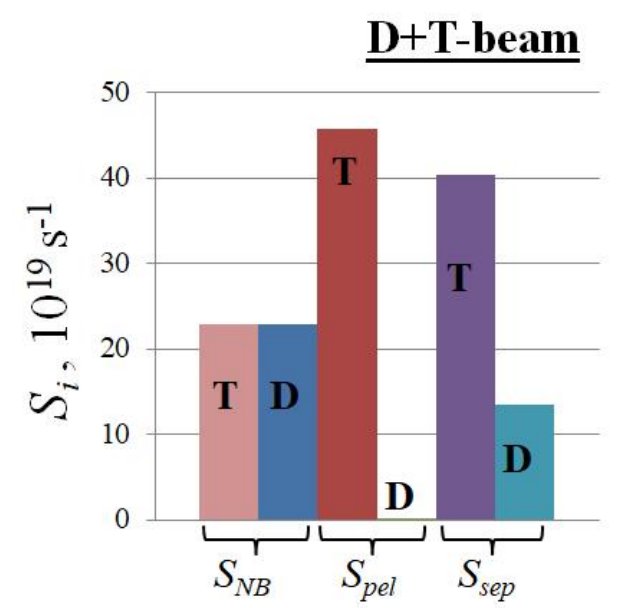

$\underline{\text { D-beam }}$

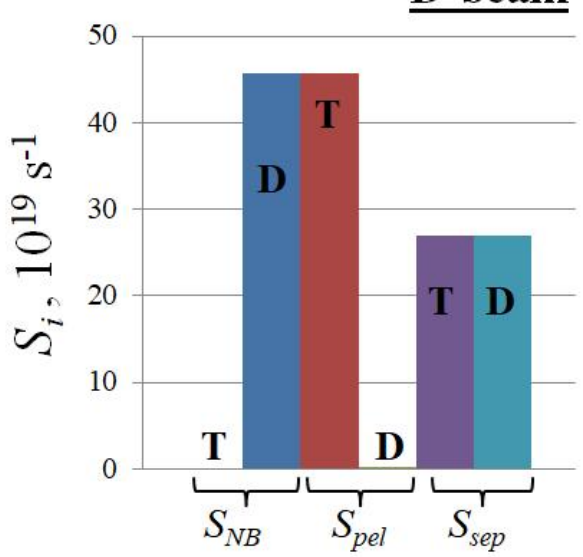

(a)

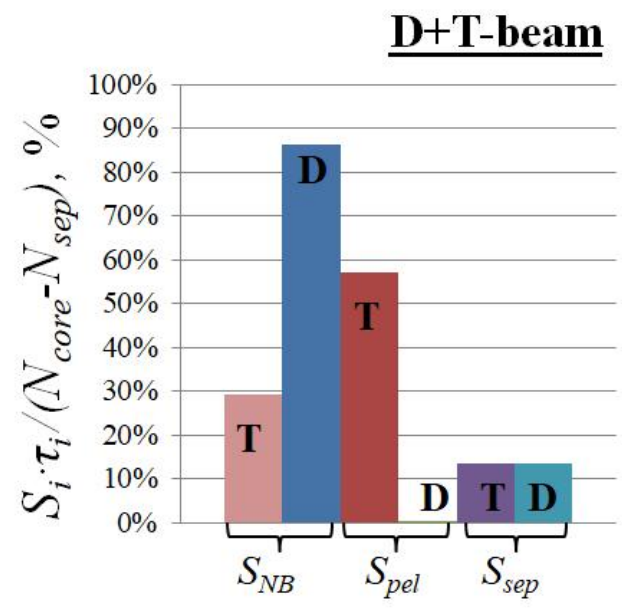

$\underline{\text { D-beam }}$

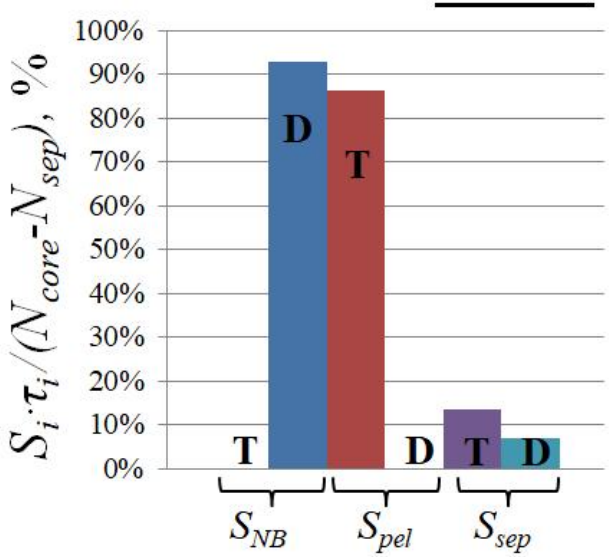

(b)

Figure 4. (a) Sources of deuterium and tritium in the plasma core, originating from the neutral beam $S_{N B}$, pellet injection $S_{\text {pel }}$, and divertor region $S_{\text {sep }}$ for the $\mathrm{D}+\mathrm{T}$ (top) and D (bottom) beams. The absolute values of the particle flux sources are given. $n_{e}=8.5 \times 10^{19} \mathrm{~m}^{-3}, D / \chi_{e}=0.4$. For the $\mathrm{D}+\mathrm{T}$ and $\mathrm{D}$ beams, $f_{\text {core }}{ }^{T}=0.75$ and 0.5 , respectively. (b) Fractions of particles from different sources with allowance for their lifetimes.

For both types of beams, the $S_{\text {neut }}$ intensities as functions of the $n_{e}$ and $D / \chi_{e}$ parameters are shown in Figure 5. In this case, the fractions of tritium are maximal, $f_{\text {core }}{ }^{T}=f_{\text {core }}{ }^{T}(\max )$, which provides the maximum possible neutron yield in the entire range of the parameters under consideration. It can be seen that the dependencies of the $S_{\text {neut }}$ intensities on the parameters are different for the scenarios with the $\mathrm{D}+\mathrm{T}$ and $\mathrm{D}$ beams (see Figure $5 \mathrm{a}, \mathrm{b}$ ). In this case, in the range of the tritium fractions $f_{\text {core }}{ }^{T}(\max )>0.5$, the ranges of neutron yield variation turn out to be comparable for both types of beams. However, the maximum values are reached in different ranges of the $n_{e}$ and $D / \chi_{e}$ parameters. For the $\mathrm{D}$ beam, the $S_{\text {neut }}$ intensity is noticeably lower compared with the D + T beam in the range of parameters where $f_{\text {core }}{ }^{T}(\max )<0.5$. This is an indirect consequence of the fact that the dependence $S_{\text {neut }}\left(f_{\text {core }}{ }^{T}\right)$ shown in Figure 2 for the central point of the parameter plane $\left(n_{e}\right.$, $\left.D / \chi_{e}\right)$ is steeper for the $\mathrm{D}$ beam than for the $\mathrm{D}+\mathrm{T}$ beam. 


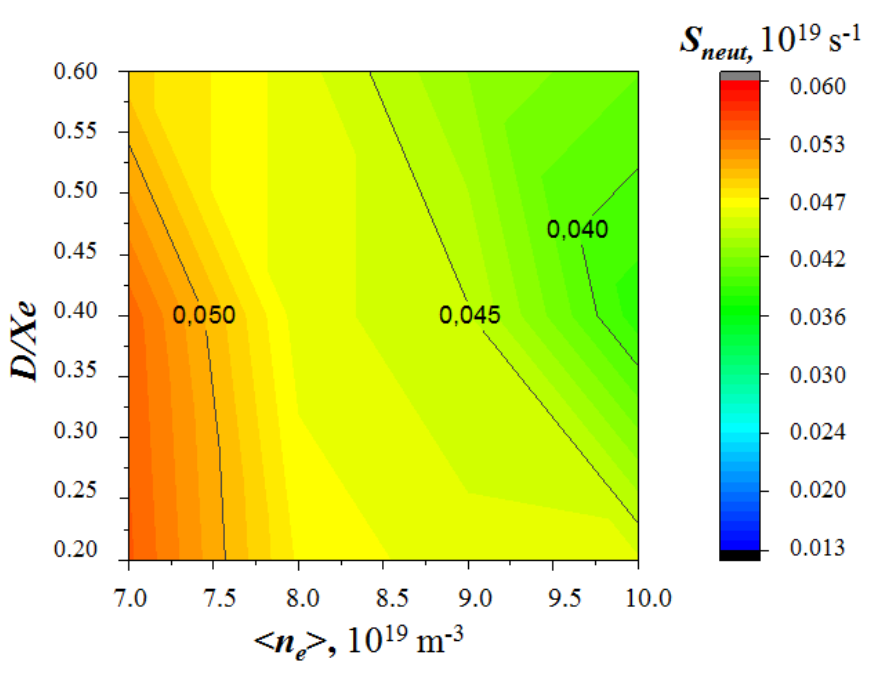

(a)

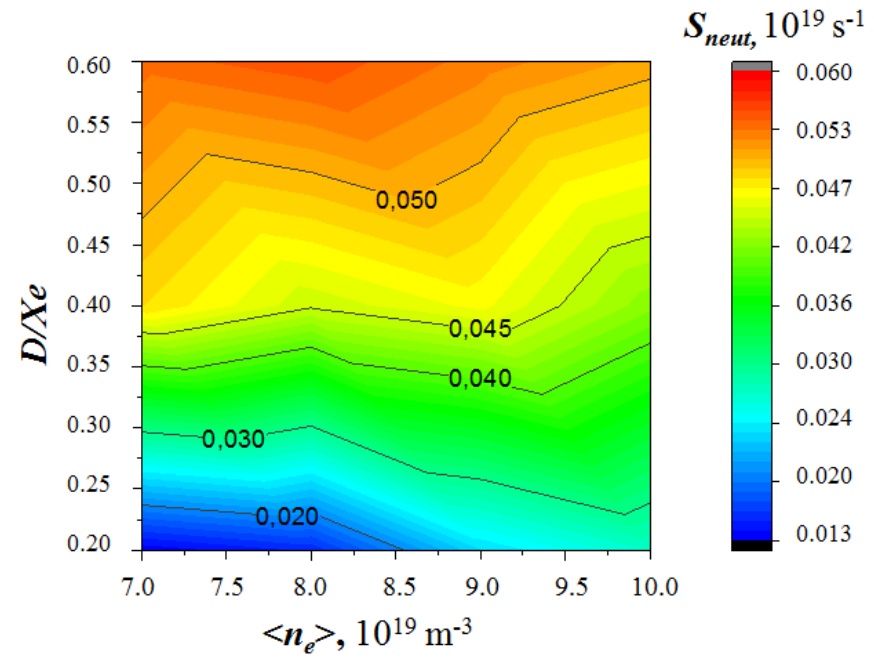

(b)

Figure 5. Neutron intensity $S_{\text {neut }}$ as a function of the $n_{e}$ and $D / \chi_{e}$ parameters for the (a) $\mathrm{D}+\mathrm{T}$ and (b) $\mathrm{D}$ beams. The scale on the right shows the correspondence of the color and $S_{\text {neut }}$ values.

The amounts of tritium accumulated at the site as functions of the $n_{e}$ and $D / \chi_{e}$ parameters are shown in Figure 6. For the $\mathrm{D}+\mathrm{T}$ beam, the simulations of the operating regimes show that the amount of tritium accumulated in the FC of the facility is quite noticeable; nevertheless, it was lower than that estimated previously in [4]. One can see that the accumulation of tritium in the FC depends directly on the fraction of tritium $f_{\text {core }}{ }^{T}$. For the $\mathrm{D}$ beam, the total amount of tritium turns out to be lower than for the $\mathrm{D}+\mathrm{T}$ beam. Primarily, this is due to the absence of tritium in the neutral beam injectors (in the case of the $\mathrm{D}+\mathrm{T}$ beam, each of them accumulates up to $100 \mathrm{~g}$ of $\mathrm{T}_{2}$ ), as well as due to the smaller amount of tritium in the hydrogen isotope separation system.

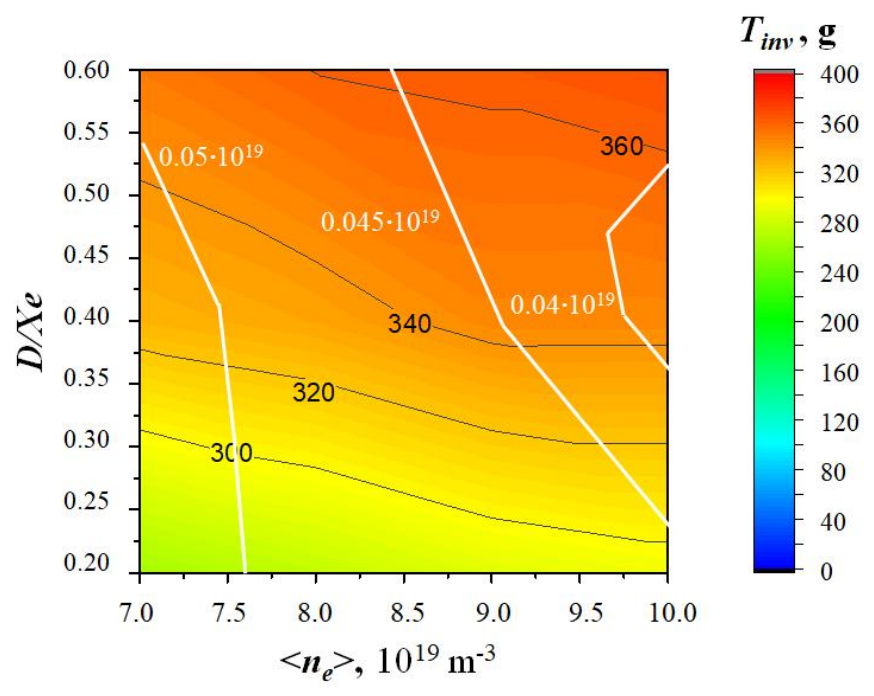

(a)

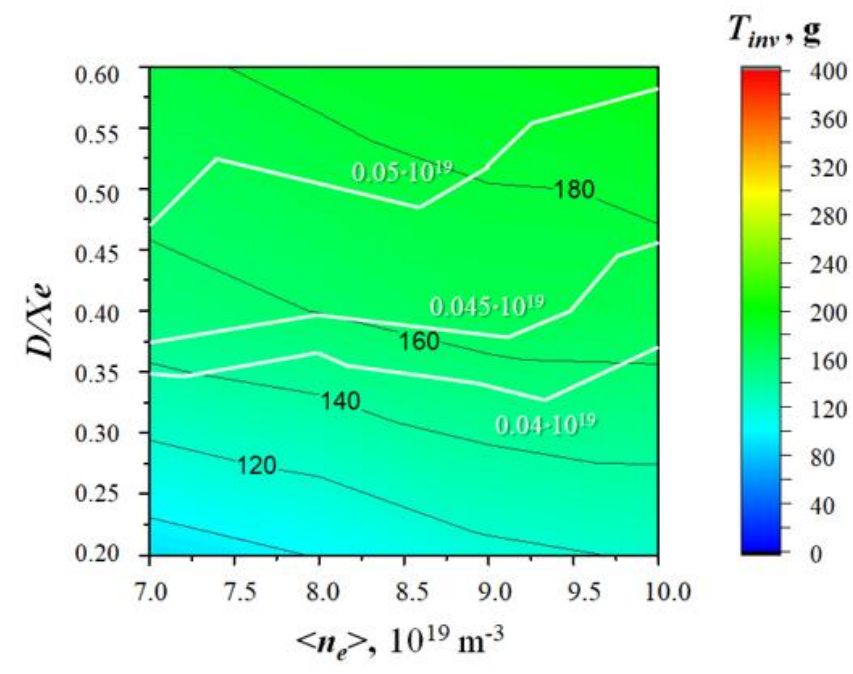

(b)

Figure 6. Amount of tritium at the site as a function of the $n_{e}$ and $D / \chi_{e}$ parameters for the (a) $\mathrm{D}+\mathrm{T}$ and (b) $\mathrm{D}$ beams. The scale on the right shows the correspondence of the color and $T_{i n v}$ values. The red lines are the level lines for the neutron intensity $S_{\text {neut }}$, shown in Figure 5, and the black lines refer to the accumulated tritium $T_{i n v}$.

By comparing Figures 5 and 6, we can decide what regimes are most reasonable from the point of view of neutron generation and the amount of accumulated tritium at the site. For the $\mathrm{D}+\mathrm{T}$ beam, to obtain the maximum neutron yield of $4.5-5.5 \times 10^{17} \mathrm{~s}^{-1}$, it would 
be required to operate in the low plasma density regimes with $n_{e}=7.0-8.5 \times 10^{19} \mathrm{~m}^{-3}$. The maximum neutron yields are reached in the range of $D / \chi_{e}=0.2-0.4$, corresponding to the good confinement of particles in the plasma. In this case, the amount of tritium accumulated in the FC can vary from 260 to $370 \mathrm{~g}$. In the regimes with the highest neutron yields, the amount of accumulated tritium is $260-340 \mathrm{~g}$. For the $\mathrm{D}$ beam, on the contrary, a maximum neutron yield of 4.5-5.5 $\times 10^{17} \mathrm{~s}^{-1}$ is observed over the entire density range under consideration, $n_{e}=7.0-10.0 \times 10^{19} \mathrm{~m}^{-3}$, in the regimes with not very good particle confinement, $D / \chi_{e}=0.4-0.6$. The amount of tritium in the FC ranges from 90 to $200 \mathrm{~g}$. At the same time, in the range of $f_{\text {core }}{ }^{T}(\max )<0.5$, the use of the D beam may result in obtaining low neutron yields (2-4 times lower than the maximum one). However, this allows operating at the minimum tritium content at the site. For convenience, in Figure 5, the white lines indicate the levels of equal intensity for the corresponding distribution of the $S_{\text {neut }}$ intensity.

\section{Conclusions}

A consistent approach was taken in simulations of the operating regimes of the FNSST facility. The simulations were performed using the SOLPS4.3 and ASTRA codes for different bulk plasma densities and diffusion particle diffusivities. For the cases of using the heating beams consisting of $\mathrm{D}+\mathrm{T}$ or pure $\mathrm{D}$ atoms at the compact neutron source FNS-ST, the dependence of the FNS neutron yield on the fraction of tritium in the bulk plasma was analyzed for the first time. The consistent modeling using the FC-FNS code made it possible to calculate the required fluxes of the fuel components into the plasma, supplied by different injection systems (pellet injectors and fast atomic beams). For both types of the heating beams, in the window of the working parameters $n_{e}$ and $D / \chi_{e}$, the maximum possible fraction of tritium in the bulk plasma was calculated, at which all deuterium particles were injected into the plasma with the beam. For the scenarios of using the $\mathrm{D}+\mathrm{T}$ or $\mathrm{D}$ beams, the neutron yields were calculated as functions of the $n_{e}$ and $D / \chi_{e}$ parameters. For the regimes with the maximum neutron yields, the accumulation of tritium at the site (in the fuel cycle) was calculated for different types of heating beams.

It was shown that for the $\mathrm{D}+\mathrm{T}$ beams, the maximum neutron yield of $4.5-5.5 \times 10^{17} \mathrm{~s}^{-1}$ could be obtained at the bulk plasma density of $n_{e}=7.0-8.5 \times 10^{19} \mathrm{~m}^{-3}$. The maximum neutron yields were reached in the range of $D / \chi_{e}=0.2-0.4$, corresponding to the good confinement of particles in the plasma. In this case, the amount of tritium accumulated in the FC of the facility is expected to range from 260 to $370 \mathrm{~g}$, including up to $150 \mathrm{~g}$ accumulated in the neutral beam injectors.

For the D beam, the maximum neutron yield of $4.5-5.5 \times 10^{17} \mathrm{~s}^{-1}$ corresponded to the density range of $n_{e}=7.0-10.0 \times 10^{19} \mathrm{~m}^{-3}$ in the regimes with not very good particle confinement, $D / \chi_{e}=0.4-0.6$. The expected amount of tritium in the FC ranged from 90 to $200 \mathrm{~g}$. At the same time, in the range of the tritium fraction $f_{\text {core }}{ }^{T}(\max )<0.5$, the use of the $\mathrm{D}$ beam may result in low neutron yields (2-4 times lower than the maximum one), although it provides the minimum tritium content at the facility site.

Based on the above estimates, we conclude that in the density range of $n_{e}=7.0-10.0 \times 10^{19} \mathrm{~m}^{-3}$, the use of the $\mathrm{D}$ beam is more reasonable. First of all, this is due to the lower tritium inventory in the FC, technical advantages associated with the absence of tritium in the injectors, and the corresponding less strict safety requirements for the operation of the injection system for plasma heating. In this case, the neutron intensities comparable in magnitude with the maximum ones (which is the priority issue for the fusion neutron source) for the $\mathrm{D}+\mathrm{T}$ beam can be obtained in a wider range of plasma densities. We note that the range of parameters favorable for using the $\mathrm{D}$ beam corresponds to somewhat worse confinement of particles in the bulk plasma, which reduces the $\mathrm{D}$ ion density in the core and, correspondingly, increases $f_{\text {core }}{ }^{T}$ (max). 
Author Contributions: FC-FNS calculations, S.A.; ASTRA calculations, A.D.; SOLPS4.3 calculations, A.K.; writing-original draft preparation, S.A.; writing-review and editing, S.A., A.D. and A.K.; project administration, S.A. All authors have read and agreed to the published version of the manuscript.

Funding: This work was supported in part by the Russian Science Foundation (project no. 18-72-10162).

Institutional Review Board Statement: Not applicable.

Informed Consent Statement: Not applicable.

Acknowledgments: The simulations with the SOLPS4.3 code were performed using computing resources of the federal collective usage center Complex for Simulation and Data Processing for Mega-science Facilities at NRC "Kurchatov Institute", http:/ / ckp.nrcki.ru/.

Conflicts of Interest: The authors declare no conflict of interest. The funders had no role in the design of the study; in the collection, analysis, or interpretation of data; in the writing of the manuscript, or in the decision to publish the results.

\section{References}

1. Kuteev, B.; Azizov, E.; Bykov, A.; Dnestrovsky, A.; Dokuka, V.; Gladush, G.; Golikov, A.; Goncharov, P.; Gryaznevich, M.P.; Gurevich, M.; et al. Steady-state operation in compact tokamaks with copper coils. Nucl. Fusion 2011, 51, 073013. [CrossRef]

2. Kuteev, B.V.; Alexeev, P.; Chernov, V.; Chukbar, B.; Bykov, A.; Frolov, A.; Golikov, A.; Goncharov, P.; Gryaznevich, M.; Gurevich, M.; et al. Conceptual design requirements and solutions for MW-range fusion neutron source FNS-ST. In Proceedings of the 24th IAEA Fusion Energy Conference, San Diego, CA, USA, 8-13 October 2012; p. 462. Available online: http:/ /www-naweb.iaea. org/napc/physics/FEC/FEC2012/html/proceedings.pdf (accessed on 15 August 2021).

3. Golikov, A.A.; Kuteev, B.V. Selection of parameters of the stationary discharge regime in the compact tokamak. Probl. At. Sci. Technol. Ser. Thermonucl. Fusion 2010, 2, 50-58.

4. Anan'ev, S.S.; Spitsyn, A.; Kuteev, B.; Shirnin, P.N.; Kazakovsky, N.T.; Cherkez, D. Concept of DT fuel cycle for a fusion neutron source. Fusion Sci. Technol. 2015, 67, 241-244. [CrossRef]

5. Dnestrovskij, A.Y.; Golikov, A.A.; Kuteev, B.V.; Khairutdinov, R.R.; Gryaznevich, M.P. Studies of stationary operating regime of the tokamak-based neutron source. Probl. At. Sci. Technol. Ser. Thermonucl. Fusion 2010, 4, $26-35$.

6. Executive Order of the President of the Russian Federation dated 04.16.2020 No. 270. On the Development of Technology, Technology and Research in the Field of Atomic Energy Use in the Russian Federation. 2020. Available online: http:/ / www. kremlin.ru/acts/bank/45436 (accessed on 15 August 2021).

7. Kuteev, B.V.; Goncharov, P.R. Fusion-fission hybrid systems: Yesterday, today, and tomorrow. Fusion Sci. Technol. 2020, 76, 836-847. [CrossRef]

8. Kuteev, B.; Azizov, E.; Alexeev, P.; Ignatiev, V.; Subbotin, S.; Tsibulskiy, V. Development of DEMO-FNS tokamak for fusion and hybrid technologies. Nucl. Fusion 2015, 55, 073035. [CrossRef]

9. Shpanskiy, Y. DEMO-FNS project team progress in the design of the DEMO-FNS hybrid facility. Nucl. Fusion 2019, 59, 076014. [CrossRef]

10. Ananyev, S.S.; Ivanov, B.V.; Spitsyn, A.V.; Kuteev, B.V. Development of a candidate technology development program for the main DEMO-FNS fuel cycle systems. In Proceedings of the 31st Symposium on Fusion Technology (SOFT2020), Dubrovnik, Croatia, 20-25 September 2020.

11. Ananyev, S.; Spitsyn, A.; Kuteev, B. Concept of DT fuel cycle for a fusion neutron source DEMO-FNS. Fusion Eng. Des. 2016, 109-111, 57-60. [CrossRef]

12. Ananyev, S.S.; Ivanov, B.V.; Kuteev, B.V. Analysis of promising technologies of DEMO-FNS fuel cycle. Fusion Eng. Des. 2020, 161, 111940. [CrossRef]

13. Dlougach, E.D.; Panasenkov, A.A.; Kuteev, B.V.; Filimonova, E.A. Neutral beam current ratio in the neutron source FNS-ST. Probl. At. Sci. Technol. Ser. Thermonucl. Fusion 2010, 44, 100-106.

14. Panasenkov, A.A.; Ananyev, S.S.; Dlougach, E.D.; Kuteev, B.V. Analysis of the setup and parameters of the FNS-ST tokamak fast atom injector. Probl. At. Sci. Technol. Ser. Thermonucl. Fusion 2021, 44, 86-99.

15. Ananyev, S.S.; Dnestrovskij, A.Y.; Kukushkin, A.S.; Spitsyn, A.V.; Kuteev, B.V. Simulation of fuel flows in injection systems of Demo-FNS hybrid facility involving coupled modeling of the core and divertor plasmas. Phys. At. Nucl. 2020, 83, $1101-1115$. [CrossRef]

16. Dnestrovskiy, A.Y.; Kukushkin, A.S.; Kuteev, B.V.; Sergeev, V.Y. Integrated modelling of core and divertor plasmas for the DEMO fusion neutron source hybrid facility. Nucl. Fusion 2019, 59, 096053. [CrossRef]

17. Kuteev, B.V.; Bykov, A.S.; Dnestrovskij, A.Y.; Golikov, A.A.; Goncharov, P.R.; Gryaznevich, M.; Gurevich, M.I.; Ivanov, A.A.; Khairutdinov, R.R.; Khripunov, V.I.; et al. Key physics issues of a compact tokamak fusion neutron source. In Proceedings of the 23rd IAEA Fusion Energy Conference, Daejeon, Korea, 11 October 2010; pp. 11-16. Available online: http:/ / www-naweb.iaea. org/napc/physics/FEC/FEC2010/html/node437.htm\#85057 (accessed on 15 August 2021). 
18. Hemsworth, R.; Esch, H.D.; Krylov, A.; Panasenkov, A.; Tanga, A. A neutral beam injector for fuelling a fusion device with atomic tritium. In Proceedings of the 9th International Conference on Tritium Science and Technology (TRITIUM 2010), Nara, Japan, 24-29 October 2010.

19. Aymar, R. Technical basis for the ITER-FEAT outline design. In ITER EDA Documentation Series, No. 19; IAEA: Vienna, Austria, 2000.

20. Ananyev, S.S.; Spitsyn, A.; Kuteev, B.V. Electronic model FC-FNS of the fusion neutron source DEMO-FNS fuel cycle and modeling hydrogen isotopes flows and inventories in fueling systems. Fusion Eng. Des. 2019, 138, 289-293. [CrossRef]

21. Ananyev, S.S.; Dnestrovskij, A.Y.; Kukushkin, A.S.; Spitsyn, A.V.; Kuteev, B.V. Architecture of Fuel systems of hybrid facility DEMO-FNS and algorithms for calculation of fuel flows in the FC-FNS model. Fusion Sci. Technol. 2020, 76, 503-512. [CrossRef]

22. Kukushkin, A.; Pacher, H.; Kotov, V.; Pacher, G.; Reiter, D. Finalizing the ITER divertor design: The key role of SOLPS modeling. Fusion Eng. Des. 2011, 86, 2865-2873. [CrossRef]

23. Pereverzev, G.V.; Yushmanov, P.N. ASTRA: Automated System for Transport Analysis in a Tokamak; Report ID 282186; Max-PlanckInstitut fur Plasmaphysik: Garching, Germany, 2002; 147p.

24. Pacher, H.; Kukushkin, A.; Pacher, G.; Kotov, V.; Pitts, R.; Reiter, D. Impurity seeding in ITER DT plasmas in a carbon-free environment. J. Nucl. Mater. 2015, 463, 591-595. [CrossRef]

25. Ananyev, S.; Dnestrovsky, A.; Kukushkin, A. Integrated modeling of fuel flows in the plasma and in the injection and pumping systems for the demo-fns fusion neutron source. Probl. At. Sci. Technol. Ser. Thermonucl. Fusion 2020, 43, 96-109. [CrossRef]

26. Dnestrovskij, A.Y.; Kukushkin, A.S.; Kuteev, B.V.; Sergeev, V.Y. Helium ash removal in DEMO-FNS. In Proceedings of the 28th IAEA Fusion Energy Conference, Saint Petersburg, Russia, 10-15 May 2021; Paper TH/P1-22. Available online: https: //nucleus.iaea.org/sites/fusionportal/Shared\%20Documents/FEC\%202020/fec2020-preprints / preprint1124.pdf (accessed on 15 August 2021).

27. Erba, M.; Cherubini, A.; Parail, V.V.; Springmann, E.; Taroni, A. Development of a non-local model for tokamak heat transport in L-mode, H-mode and transient regimes. Plasma Phys. Control. Fusion 1997, 39, 261-276. [CrossRef]

28. Erba, M.; Aniel, T.; Basiuk, V.; Becoulet, A.; Litaudon, X. Validation of a new mixed Bohm/gyro-Bohm model for electron and ion heat transport against the ITER, Tore Supra and START database discharges. Nucl. Fusion 1998, 38, 1013-1028. [CrossRef]

29. Nguyen, C.N.; Bateman, G.; Kritz, A.H.; Akers, R.; Byrom, C.; Sykes, A.; Team, M. Integrated predictive modeling simulations of the Mega-Amp Spherical Tokamak. Phys. Plasmas 2002, 9, 3930-3937. [CrossRef]

30. Polevoi, A.; Shimada, M.; Sugihara, M.; Igitkhanov, Y.; Mukhovatov, V.; Kukushkin, A.; Medvedev, S.; Zvonkov, A.; Ivanov, A. Requirements for pellet injection in ITER scenarios with enhanced particle confinement. Nucl. Fusion 2005, 45, 1451-1456. [CrossRef]

31. Valovič, M.; Meyer, H.; Akers, R.; Brickley, C.; Conway, N.; Cunningham, G.; Kirk, A.; Lloyd, B.; Patel, A.; Taylor, D.; et al. Energy and particle confinement in MAST. Nucl. Fusion 2005, 45, 942-949. [CrossRef]

32. Pankin, A.; McCune, D.; Andre, R.; Bateman, G.; Kritz, A. The tokamak Monte Carlo fast ion module NUBEAM in the National Transport Code Collaboration library. Comput. Phys. Commun. 2004, 159, 157-184. [CrossRef] 